DE LA PUBLICIDAD DE

"BIEN PÚBLICO" A LA

\section{PUBLICIDAD SOCIAL EN}

ARGENTINA

\section{José Luis Taricco}

Profesor de Comunicación en Publi-

cidad y Propaganda

Universidad Nacional de Córdoba, Argentina.

Email: jltaricco@yahoo.com.ar

\section{Resumen}

En la Argentina el fenómeno de la denominada hoy publicidad social tiene una importante historia. En los últimos años ha recibido nuevos aportes y conceptos, de la mano de los procedimientos del Marketing Social y de la denominada Responsabilidad Social Empresaria.

No es menos cierto que el contexto de crecimiento económico(al menos hasta ahora) de los últimos años, volvió a dar impulso a las campañas sociales, tanto desde el sector privado apoyando a las ONG, cómo del propio Estado Nacional que incluyo muchos temas sociales en las estrategias de comunicación oficial.

\section{Palabras clave}

Publicidad de Bien Público, Responsabilidad Social Empresaria, Publicidad Oficial, Comunicaciones Globales, Estrategias Múltiples, Publicidad Social, Desarrollo

Sustentable

Key Words

Non profit advertising, Corporate Social

Responsibility, Goverment Advertising, Global Communications, Multiple Creative Strategies, Social Advertising-Sustainable development

Abstract

In Argentina the phenomena of the today called social advertising has an important background. In the last years it has received new contributions and concepts, along with the procedures of Social Marketing and she so

called Corporate Social Responsability.

It is also true that the context of economic grouth (at least up to now) of the last years again gave an impulse to the social campaigns both from the private sector supporting the NGO, as well as from the state, which included many social issues in its strategies of communication. 


\section{Introducción}

En la Argentina el fenómeno de la denominada hoy publicidad social tiene una importante historia que comienza en la década de los años 60 con la denominación de Publicidad de "Bien Público".

En los últimos años a recibido nuevos aportes y conceptos, de la mano de los procedimientos del Marketing Social y de la denominada Responsabilidad Social Empresaria.

No caben dudas de que aumentó considerablemente la inversión, mucho más la pública que la privada, en los rubros de publicidad en general y de publicidad social en particular de la mano de un crecimiento económico sustentable en los últimos años.

También es cierto que las corporaciones empresarias se están agrupando en torno al tema de la Responsabilidad Social Empresaria (RSE), y gestionan cada vez con mayor rigurosidad sus acciones hacia los problemas “sociales”, más allá de sus productos y servicios.

Por otro lado a los problemas sociales de cada país en particular se suman los problemas "globales", tanto ambientales como de agotamiento de recursos considerados estratégicos.

Todo ello sumado a una prominencia del mercado por sobre el rol de los estados, está dando el contexto para asumir desde los procesos de comunicación social en general y desde la publicidad en particular, un mayor compromiso con la humanidad.

\section{Objetivos}

El objetivo de este ensayo es destacar el aumento de la inversión en el campo de la comunicación social desde el Estado-Nación como desde las empresas privadas y las ONG, en el denominado proceso de "publicidad social" o de "bién público".

Se destaca, también, la necesidad de la toma de conciencia de los organismos socia- les, privados-estatales - organismos intermedios, en torno al compromiso público desde la comunicación para contribuir a disminuir o resolver la multiplicidad de temas sociales que generan injusticias, inequidades y desequilibrios sociales. 


\section{Metodología}

Es un ensayo de aproximación a la problemática en el marco de un trabajo de investigación sobre modos y sistemas de comunicación de las ONG de Córdoba, Argentina, en convenio con la Universidad Rey Juan Carlos de España.

\section{Los Pioneros}

Desde 1960 el Consejo Publicitario Argentino se oriento específicamente a la comunicación de bien público como ellos mismo lo definen:

\section{¿Qué es la Comunicación de Bien Publico y cuál es la misión del Conse- jo Publicitario?}

La comunicación de Bien Público es definida como la Publicidad que se dedica al Bien Público. La mayoría de los avisos de bien público utilizan espacios cedidos gratuitamente por los medios. El objetivo de estos mensajes es generar conciencia sobre temas socialmente importantes para cambiar actitudes y comportamientos de la gente y, así , lograr una sociedad mejor.

Este modelo de comunicación surgió en Estados Unidos durante la segunda Guerra Mundial, para conseguir soldados. El Consejo Publicitario Argentino, entidad priva$\mathrm{da}$, sin fines de lucro, fue fundado el 21 de septiembre de 1960, por los tres sectores que integran la Comunicación Publicitaria: Empresas, Agencias de Publicidad, Medios de Comunicación.

Su misión es, desarrollar Campañas de Comunicación de problemas relevantes de la comunidad, para crear conciencia e inducir a la acción para solucionarlos. Este es el objetivo fundamental del Consejo, desarrollar y difundir las campañas hasta lograr resultados.

El Consejo Publicitario realiza Campañas de Bien Publico nacionales, que colaboran con distintas ONG, no siguen un discurso político, no son comerciales, ni religiosas, se llevan a cabo, en principio, mediante la donación de creatividad, recursos y medios de sus miembros

\section{¿Marketing comercial, marketing social y campañas de bien publico?}


El marketing social engloba las campañas de bien público pero no son términos intercambiables. El concepto de marketing social data de 1971 y lo utilizaron Kotler y Zaltman para aplicar los postulados y técnicas del marketing comercial, en campañas de bien público o la difusión de ideas que beneficien a la sociedad. Desde entonces este concepto se utiliza para referirse a promover acciones o prácticas sociales en una determinada comunidad.

A diferencia del marketing comercial no deben guiarse por la orientación a venta que muchas veces aparecen en programas de comunicación. La estrategia del marketing social, comienza concentrándose en el público objetivo que será destinatario del programa de marketing social, es decir será el cliente.

¿Cómo se generan las Campañas del Consejo Publicitario?
Las Campañas se realizan con el apoyo desinteresado de los asociados, en las diferentes áreas:

- Empresas Anunciantes que aportan recursos materiales.

- Agencias de Publicidad que desarrollan estrategias creativas.

- Medios de Comunicación que ceden gratuitamente espacios, para la difusión de los mensajes.

Cada campaña es realizada con la colaboración de ONG expertas en el tema que se trate, quienes colaboran en la medida de sus posibilidades a cubrir los costos de producción y distribución.

Mediante Comisiones de socios se desarrollan la campañas, con el apoyo de investigación, planificación de medios y actividades de prensa. (-1)

\section{La inversión del Estado}

En la Argentina de los últimos años se incremento de modo muy significativo la inversión por tanto el gasto en publicidad desde el estado. Gran parte de este fue a Campañas de temas / problemas sociales.

El aumento en el gasto en publicidad oficial podría explicarse debido a las subas en los costos de las tarifas, derivación del fenómeno económico de la inflación; por un incremento de los medios en los que pautar y/o por un aumento en las necesidades comunicacionales del gobierno. El secretario de Medios de la Nación, Enrique Albistur, sostuvo en una entrevista con el diario La Nación que el fenómeno inflacionario era 
una de las causas del aumento en el gasto del gobierno en publicidad oficial para 2007, junto al impulso de la campaña de "marca país".

El constante crecimiento interanual del presupuesto supera ampliamente los índices de inflación oficiales. Así, mientras que en 2004 la inflación fue del 6,1\%, en dicho año el aumento del gasto fue del orden del $115,73 \%$ respecto del año anterior. Este es un parámetro que se repitió en los años siguientes.

\section{Crecimiento interanual del gasto en publicidad oficial}

$2003 * 46.267 .906,003,7$

2004*99.815.380,00 6,1 115,73\%

2005 127.462.075,00 12,3 27,70\%

2006 209.638.868,61 9,8 64,47\%

(-2) Fuente: $A D C$ en base a las plani-

Ilas de inversión publicitaria proporcionadas por la Secretaría de Medios de la Nación.

\section{INVERSIÓN POR CAMPAÑAS}

La publicidad oficial debe entenderse como un canal de comunicación entre el

Estado y la población, que sirva para difundir información clara, objetiva, necesaria,

útil y relevante para la ciudadanía. En ese sentido, es importante conocer y controlar a qué campañas publicitarias y avisos han sido efectivamente destinados los fondos públicos.

5 "Primer semestre de 2007: las primeras veinte campañas

Según la información provista por TELAM ( Agencia de Noticias del Estado)a través de la Secretaría de Medios de la Nación, la principal campaña publicitaria impulsada por ese organismo en la primera mitad de 2007 fue la de “Accidentes de Tránsito”, del Ministerio del Interior, que requirió una inversión de 15.726.553,94 pesos.

En segundo lugar se ubicó la campaña "Alcoholemia" de la Secretaría de Prevención del Narcotráfico, con 15.046.327,29 pesos y en tercer lugar la campaña "Antitabaco", del Ministerio de Salud, que requirió 12.329.105,86 pesos. 
Las principales veinte campañas de este primer semestre fueron:

Nombre de Campañas y Avisos Organismo Total Gastado

“Accidentes de Tránsito” Ministerio del Interior 15.726.553,94

“Alcoholemia” Secretaría de Prevención del Narcotráfico 15.046.327,29

“Antitabaco" Ministerio de Salud 12.329.105,86

“Mercado" Ministerio de Economía 8.136.555,45

"Visitas" Presidencia de la Nación 6.452.855,7

“Trabajo” Ministerio de Trabajo 6.287.931,58

“Innovar” Ministerio de Educación 5.864.782,07

“Servicio Doméstico" AFIP 5.632.150,92

“Ganancias” AFIP 5.097.585,44

"Factura en Mano" AFIP 5.064.021,11

"Licitaciones" Varios 4.814.186,59

“Secundario Obligatorio" Ministerio de Educación 4.799.871,87

“Transito" Ministerio del Interior 4.748.846,65

"Institucionales" Varios 4.647.663,35

“Aduana Segura” AFIP 4.300.202,87

“Marca Argentina / Pais” Presidencia de la Nación 4.062.973, 55

“Genéricos” Ministerio de Salud 4.013.362,27

“Consumo” Secretaría de Agricultura 3.652.459,54

"Desarmaderos" Ministerio de Justicia 3.441.996,12

“Declaración Proforma” AFIP 2.991.247,58

Fuente: $A D C$ en base a las planillas de inversión publicitaria proporcionadas por la Secretaría de Medios de la Nación. Incluye únicamente clientes centralizados

\section{La Inversión en Publicidad Privada}

Se observa también un importante incremento en la facturación publicitaria en todos los medios, en los últimos años en relación al crecimiento anual del país. 
CUADRO DE EVOLUCIÓN DE INVERSIÓN PUBLICITARIA 1997-2007

En miles de Pesos

\begin{tabular}{|c|c|c|c|c|c|c|c|c|c|c|c|}
\hline RUBRO & 1997 & 1998 & 1999 & 2000 & 2001 & 2002 & 2003 & 2004 & 2005 & 2006 & 2007 \\
\hline & (1) & [1) & (1) & (1) & (1) & (2) & (2) & (2) & (3) & (3) & 3 \\
\hline 1. TELEVISION & 1.311 .743 & 1.278 .154 & 1.390 .190 & 1.223 .405 & 1.050 .954 & 743.446 & 1.037 .581 & 1.289 .005 & $1.743 .94 \mathrm{E}$ & 2.237 .213 & 2.650 .061 \\
\hline 1.1. TELEVISION ABIERTA & 1.200 .526 & 1.174 .063 & 1.267 .184 & 1.088 .992 & 955.996 & 659000 & 914.91 & 1.138 .121 & 1.482 .663 & 1.866 .229 & 2225.939 \\
\hline - Capital & 952.964 & 952.545 & 1.014 .404 & 653274 & 739.730 & 528.719 & 733.329 & 909.326 & 1.177 .856 & 1.508 .21 & 1.856 .159 \\
\hline - Interior Nacional y Local & 247.562 & 221.518 & 252.780 & 235.718 & 216.266 & 130.280 & 181.582 & 228.796 & 304.805 & 358.018 & 369.780 \\
\hline 1.2 TELEVISION POR CABLE & 111.217 & 104.091 & 123.006 & 134.413 & 94.958 & 84.446 & 122.670 & 150.884 & 261.283 & 370.984 & 424.123 \\
\hline 2. MEDIOS GRAFICOS & 1.721 .866 & 1.871 .620 & 1.679 .897 & 1.371 .937 & 1.184 .067 & 842093 & 1.091 .595 & 1.367 .874 & 1.609 .876 & 1.991 .180 & 2.209 .718 \\
\hline 2.1. DIARIOS & 1.146 .680 & 1.241 .667 & 1.090 .317 & 893.962 & 808.834 & 703.569 & 899.686 & 1.107 .833 & 1.281 .576 & 1.627 .546 & 1.769 .242 \\
\hline - Capital & 1.056 .068 & 1.114 .399 & 960.654 & 760.751 & 695.473 & 608.184 & 777.713 & $956.58 \overrightarrow{7}$ & 1.104 .269 & 1.402 .691 & 1.524 .350 \\
\hline - Interior (Camp. Nac) & 90.613 & 127.279 & 129.664 & 133211 & 113.36 & 95.385 & 121.973 & 151.246 & 177.312 & 224.855 & 244.89 \\
\hline 2.2 CLASIFICADOS Y REIMATES & 312.274 & 337.928 & 283.614 & 146.457 & 104.150 & 49740 & 63.605 & 78.234 & 90.561 & 112.350 & 133.135 \\
\hline 2.3. REVISTAS & 262.911 & 292.025 & 305.969 & 331.518 & 271.084 & 88.783 & 128.304 & 181.807 & 237.739 & 251.284 & 307.342 \\
\hline 3. RADIO CAPITAL & 219.521 & 214.965 & 204.125 & 231.555 & 226.232 & 78.284 & 82.707 & 93.468 & 105.157 & 126.049 & 15284 \\
\hline 4. VIA PUBLICA & 192.864 & 206.864 & 204.147 & 228.909 & 168.501 & 116.199 & 128.035 & 188.211 & 231.211 & 348.212 & 538.878 \\
\hline 5. CINE & 49.643 & 48.532 & 54.177 & 54.575 & 43.884 & 30.280 & 36.862 & 43.497 & 56.658 & 65.700 & 75.500 \\
\hline 5. INTERNET & & & & & & 13.225 & 16.917 & 24.000 & 32.502 & 91.000 & 130.785 \\
\hline 8. PRODUCCION (4) & 318.336 & 328.226 & 324.892 & 296.392 & 256.949 & 186.037 & 233.009 & 292.782 & 368.879 & 474.700 & 591.032 \\
\hline TOTALES & 3.813 .974 & 3.948 .411 & 3.357 .428 & 3.406 .774 & 2.930 .587 & 2.009 .559 & 2.626 .706 & 3.298 .838 & 4.148 .228 & 5.334 .055 & 6.348 .815 \\
\hline
\end{tabular}

(1) FUENTE: Estimaciones de la AAAP en base a datos provistos por. Monitor de Medios Publicitarios S.A., Film Suez, LAB interactive Advertising Bureau y Alverto Scopesi y Cia. S.A.

(2) FUENTE: Estimaciones de la AAAP y la C.A.C.E.M. en base a datos provistos por. Monitor de Medios Publicitarios S.A., Bureau de Publicidad en Cable,

Bureau de Publicidad en intemet y Alberto Sccpesi y Cia. S.A. E rubro Cine es una proyección estimada en base a información de mercado

(3) FUENTE: Estimaciones de la AAAP, en base a datos provistos por. Monitor de Medios Publicitarios S.A. Film Suez, Interactive Advertising Bureau y Alberto Scopesi y Cia. S.A.

(4) El rubro Producción se estima como un porcentaje (10\%) del total de la imversión publictaria relevada, sin incluir clasificados y remates.

Fuente: Estimaciones de la AAAP, en base a datos provistos por: Monitor de Medios Publicitarios. 


\section{El Nuevo paradigma: La Responsabili- dad Social Empresarial}

A las inversiones clásicas en Publicidad se le suman en estos tiempos el agrupamiento de las empresas detrás del fenómeno denominado de Responsabilidad Social Empresaria. En Córdoba existe una ONG pionera denominada IARSE (Instituto Argentino de Responsabilidad Social Empresaria) que desempeña diversas actividades con las Empresas, entre ellas; capacitación, creación de manuales de RSE., la interconexión con organismos similares de Brasil, México y el resto del mundo.

El mundo de las empresas ha incluido rápidamente el concepto y reorientado su gasto desde las políticas de marketing y comunicación hacia acciones de neto corte social.

Los expertos sostienen que hay diversos enfoques, habría uno de corte "moral", implicaría hacer lo correcto como buen ciudadano, desde una perspectiva ética, otro enfoque esta vinculado con la "sustentabilidad”, en el largo plazo, enfatiza tutorías ambientales y comunitarias, otro enfoque esta vinculado con la "licencia para operar", y un ultimo enfoque de la "reputación" vinculado con el cuidado de la imagen, Los dos últimos están en relación a necesidades de públicos externos inmediatos. (-5)
En cualquier situación por la que optaren las empresas no cabe dudas que forma parte de las estrategias empresariales frente a un mundo que cambia vertiginosamente, en donde las condiciones sociales de los consumidores, por tanto de los ciudadanos, ya no son solo un problema de los Estados y de las organizaciones intermedias, sino también de las propias empresas, sea cual fuere su condición (privadas, cooperativas o estatales) en tanto sean productores de bienes o servicios deben involucrarse en los problemas que cada vez son mas sensibles a los imaginarios colectivos de los consumidores.

En una reciente encuesta, en una muestra realizada por la empresa MKT en la ciudad de Córdoba y a solicitud del agrupamiemto de empresas denominado Gestión Responsable, se les pregunto a los ciudadanos cuestiones como ¿Qué rol deben cumplir las empresas en la sociedad?, ¿es bueno o malo que las empresas quieran ganar dinero?, ¿ tienen que preocuparse solo por hacer negocios o también tienen que preocuparse por cuestiones sociales y ambientales?, entre otras tantas de este tono.

Estas son algunas respuestas y sus conclusiones: 
Gráficos 1 y 2: Responsabilidad Social Empresarial

Responsabilidad Social Em presaria

Hay personas que opinan que las empresas, además de preocuparse por hacer negocios, deberían preocuparse por mejorar la situación social de su comunidad. ¿Está Ud de acuerdo o en desacuerdo con esta opinión?

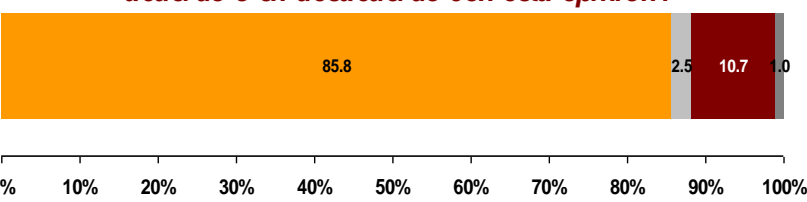

$\square$ De acuerdo $\square$ Ni de acuerdo ni en desacuerdo $\square$ En desacuerdo $\square$ NSNC
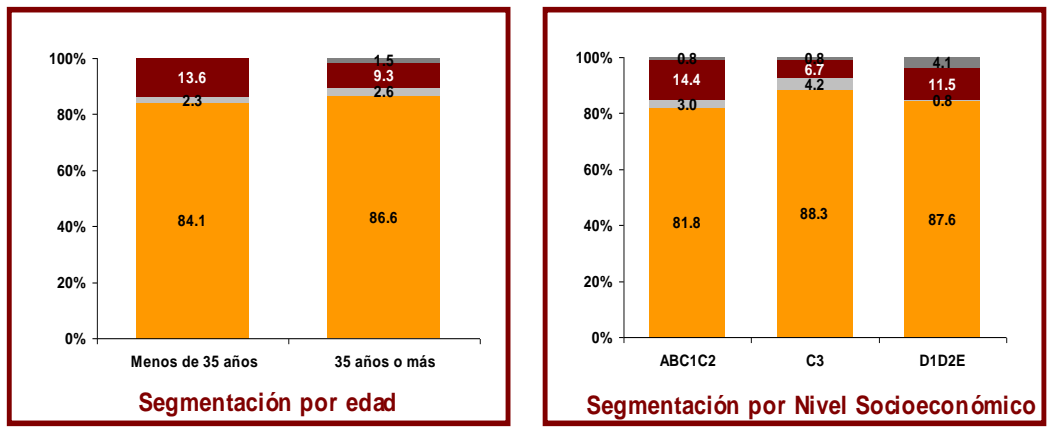

Fuente: MKT

Diapositiva No. 22

Fuente: $M K T$ 


\section{Contribución de las empresas al país}

En su opinión, ¿cuál es la principal contribución que tienen que hacer las empresas al país? Pregunta abierta. Respuestas espontáneas.

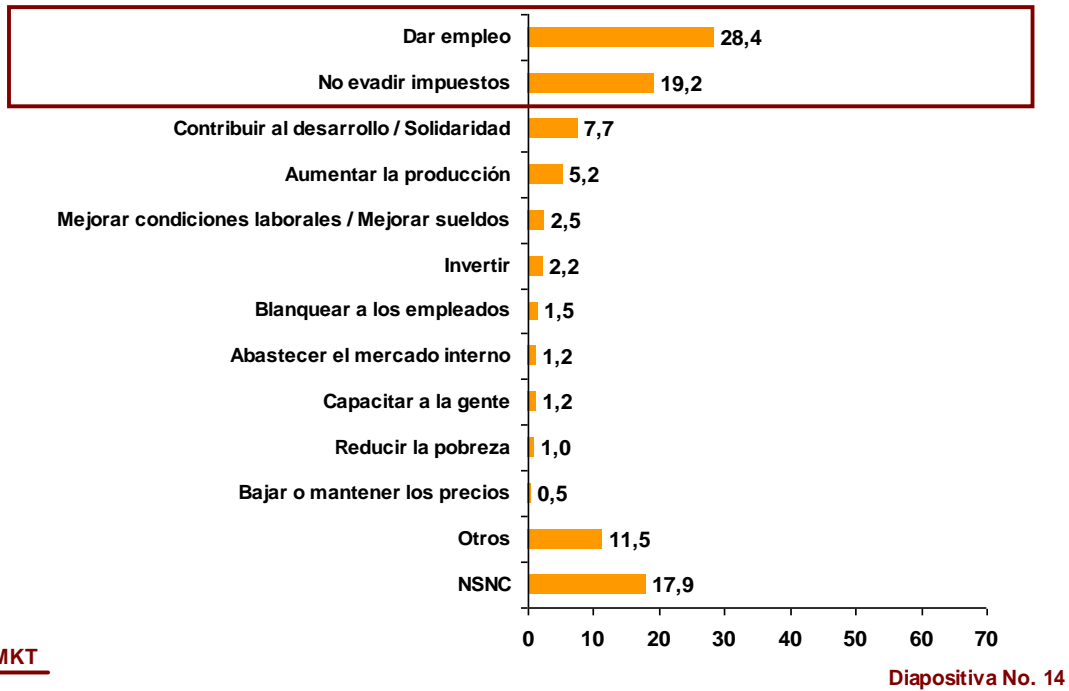

Fuente: $M K T$

\section{Síntes is de resultados}

No hay un sesgo muy marcado entre las preferencias por acciones de RSE destinadas a mejorar el medioambiente y acciones destinadas a mejorar la situación social. En efecto, el $36.2 \%$ de los cordobeses prefiere acciones de mejora social, el $33.4 \%$ prefiere acciones de mejora ambiental y al $29.7 \%$ le da igual cualquiera de las dos. Sí aparece una mayor preferencia por acciones de impacto social entre los cordobeses más jóvenes.

Poco más de la mitad de los cordobeses (52.6\%) opina que las empresas deberían comunicar sus acciones de RSE para contagiar a otras empresas, mientras que un $35.9 \%$ prefiere que las empresas, en lugar de destinar recursos a comunicar acciones de RSE, los destinen a realizar otras acciones de RSE.

\section{$(-6)$}




\section{El rol de la Publicidad Social}

Como vamos observando el mundo de las comunicaciones se transforma de modo tal que nada es lo que parece, los cambios en los sistemas de comunicación organizacional con nuevos procedimientos que plantean una revisión metodológica y conceptual, nos obligan a intentar incluir estas "nuevas prácticas” en las tradicionales e ir acompañando los tiempos de los ciudadanosconsumidores a los cambios desde las organizaciones, sean productivas o sociales.

\section{Algunas cuestiones para tener en cuenta}

Los viejos paradigmas de la comunicaciones empresariales fragmentadas (Prensa, Publicidad, RRPP, Marketing) están dando paso a nuevos paradigmas donde las comunicaciones se entienden como "integradas", o globales. En este concepto la Publicidad adquiere nuevos significados en tanto no es un proceso aislado en si mismo, sino por el contrario integrado a una estrategia global. La segunda cuestión es que el campo de las mediaciones se a diversificado enormemente (las llamadas PNT, blog, Internet, telefonía, sistemas de televisión digitales, etc.), superando ampliamente el concepto de “medios masivos de comunicación”. Cambia también el modelo de planificación de la propia publicidad que si bien es cierto define conceptos, ideas creativas y soportes diversos, lo hace en el marco de mayores "segmentaciones" de públicos, en menor tiempo. Con lo cual es común hablar de múltiples estrategias de comunicación y no solo de una.

Las condiciones de cambios de la publicidad deben ser acompañadas del análisis de los cambio de los contextos de las empresas, tanto de producción como de servicios. $\mathrm{y}$, fundamentalmente de los consumidores. Mas que ayudar a vender "tangibles" hoy lo que se venden son situaciones de vida, "intangibles", las condiciones "subjetivas "de los actores sociales definen mas la compra de un producto que sus propia realidad objetiva.

Por supuesto hay diferencias según de que producto o servicio estemos hablando.

Al desconfigurarse el rol de los productos en un mundo cada vez más simbólico, el límite entre lo privado y lo social, también se ha diluido.

Lo social ha dejado de ser un problema de los Estados y de las organizaciones Intermedias de la sociedad. Las empresas y sus coorporaciones son legitimadas desde los imaginarios sociales como articuladoras del empleo, constructora de sociabilidad, sostén de referentes familiares y sociales. En un mundo que puja, en estos días, entre 
los circuitos financieros (el dinero que genera dinero) vs. la producción industrial que genera organicidad, empleabilidad y mejor reparto de la torta, las sociedades no dudan en apoyar estas últimas, al menos desde las mayorías populares.

Nunca como en estas épocas las organizaciones empresariales estuvieron tan cerca de lo público-social. Ello exige a las empresas una mayor responsabilidad social histórica que nunca antes tuvieron.
El rol de la Publicidad Social. excede ampliamente la cuestión de la imagen, su ética o su reputación, implica un involucrase en la vida social acompañando los cambios imprescindibles para que el mundo del futuro no sea un lugar de autoextinción, no solo por los problema ambientales, sino por la justa y equitativa distribución de los recursos y de las riquezas. Nadie se salva solo en el mundo del futuro. La Publicidad definitivamente tiene que contribuir el bien social.

\section{Conclusiones}

- La inversión en publicidad social aumento de modo exponencial en los últimos años, con mas ímpetu en el Estado que en las Corporaciones Privadas, se advierte , también una mayor incidencia de ONG que logran desviar el gasto clásico de publicidad privada a sus asuntos sociales.

- Los estudios de Opinión señalan que los ciudadanos valorizan, cada vez más, el rol de las empresas como empleadoras y responsables frente a problemáticas sociales, desde las ambientales a la pobreza. etc.

- Las Empresas asociadas entre si o intermediadas por ONG cada vez mas asumen la necesidad de invertir en causas sociales y en sus entornos inmediatos. En el marco de las RSE

- La Publicidad Social no es mas que una manera de invertir en comunicación social apoyando los intentos de los organismos Estaduales, Internacionales y a las Organizaciones No Gubernamentales en problemáticas cada vez más urgentes $\mathrm{y}$ mas complejas de resolver que afectan de modo directo a millones de personas en el mundo y a la propia supervivencia del planeta.

\section{Referencias}


(-1) Datos de la Pag. WEB del Concejo Publicitario Argentino.-

(-2) y (-3) 2Fuente: ADC en base a las planillas de inversión publicitaria proporcionadas por la Secretaría de Medios de la Nación. Incluye únicamente clientes centralizados. *Los datos referentes a 2003 y 2004 corresponden a Poder Ciudadano.

(-4) FUENTE: Estimaciones de la AAAP, en base a datos provistos por: Monitor de Medios Publicitarios
S.A., Film Suez, Interactive Advertising Bureau y Alberto Scopesi

(-5) "Estrategia y Sociedad" M. Porter y M Kramer. Harvard B. Review . 2005

(-6) Estudio de opinión. Empresa MKT, y grupo de empresas Gestión Responsable- Córdoba-Argentina2007.

Cita de este artículo

Jose Luis Taricco: De la Publicidad de "Bien Público " a la Publicidad Social en Argentina. Revista Icono14 [en línea] 1 de junio de 2009, № 13. pp. 24-36. Recuperado (Fecha de acceso), de http://www.icono14.net 